\section{Global and regional brain atrophy is associated with low or retrograde facial vein flow in multiple sclerosis}

\author{
Dejan Jakimovski, ${ }^{1}$ Karen Marr, ${ }^{1}$ \\ Marcello Mancini, ${ }^{2}$ \\ Maria Grazia Caprio, ${ }^{2}$ Sirin Gandhi, ${ }^{1}$ \\ Niels Bergsland, ${ }^{1}$ Ivo Paunkoski, ${ }^{1}$ \\ Jesper Hagemeier, ${ }^{1}$ Avinash Chandra, ${ }^{1}$ \\ Bianca Weinstock-Guttman, ${ }^{3}$ \\ brain atrophy, suggests that the severity of \\ neurodegenerative process may be related \\ to hemodynamic alterations. MS patients \\ with more advanced global and regional \\ brain atrophy showed low or retrograde FV \\ volume flow.
}

Robert Zivadinov ${ }^{1,4}$

${ }^{1}$ Buffalo Neuroimaging Analysis Center, Department of Neurology, Jacobs School of Medicine and Biomedical Sciences, University of Buffalo, State University of New York, NY, USA; ${ }^{2}$ Institute of Biostructure and Bioimaging, National Research Council, Napoli, Italy; ${ }^{3}$ Jacobs Multiple Sclerosis Center, Department of Neurology, School of Medicine and Biomedical Sciences, University at Buffalo, State University of New York, Buffalo, NY, USA; ${ }^{4}$ Translational Imaging Center at Clinical Translational Science Institute, University of Buffalo, State University of New York, NY, USA

\begin{abstract}
Increased collateral facial vein (FV) flow may be associated with structural damage in patients with multiple sclerosis (MS). The objective was to assess differences in FV flow and magnetic resonance imaging (MRI)-derived outcomes in MS. The study included $136 \mathrm{MS}$ patients who underwent neck and head vascular system examination by echo-color Doppler. Inflammatory MRI markers were assessed on a 3T MRI using a semi-automated edge detection and contouring/thresholding technique. MRI volumetric outcomes of whole brain (WB), gray matter (GM), white matter (WM), cortex, ventricular cerebrospinal fluid (vCSF), deep gray matter (DGM), thalamus, caudate nucleus $(\mathrm{CN})$, putamen, globus pallidus (GP), and hippocampus were calculated. Independent $t$-test and ANCOVA, adjusted for age, were used to compare groups based on FV flow quartiles. Thirty-four MS patients with FV flow $\leq 327.8 \mathrm{~mL} / \mathrm{min}$ (lowest quartile) had significantly lower WB $(\mathrm{P}<0.001), \quad \mathrm{WM} \quad(\mathrm{P}<0.001), \quad$ thalamus $(\mathrm{P}=0.004), \quad$ cortex $\quad(\mathrm{P}=0.004), \quad \mathrm{GM}$ $(\mathrm{P}=0.004)$, DGM $(\mathrm{P}=0.008)$, hippocampus $(\mathrm{P}=0.005)$, and GP volumes $(\mathrm{P}=0.044)$ compared to 102 patients with FV flow of $>327.8 \mathrm{~mL} / \mathrm{min}$ (higher quartiles). There
\end{abstract}

were no differences in T1-, T2- and gadolinium-enhancing lesion volumes between the quartile groups.

The lack of an association between FV blood flow and inflammatory MRI measures in MS patients, but an association with

\section{Introduction}

Multiple sclerosis (MS) is a chronic autoimmune-mediated demyelinating disorder of the central nervous system (CNS). However, a neurodegenerative component is being increasingly recognized as an important contributor to the disease pathophysiology. Global and regional brain atrophy in MS has been strongly associated with both physical and cognitive decline. ${ }^{1}$

An emerging vascular hypothesis has sparked intense research regarding the anatomy and physiology of the vascular system in association with the disease pathophysiology. ${ }^{2}$ A variety of invasive and non-invasive imaging modalities have been used to describe changes in the venous system. ${ }^{3}$ Despite the advantages and disadvantages of each technique, color Doppler ultrasound allows real-time, dynamic examination of both the structural and hemodynamic properties of the venous system and remains a valuable diagnostic test. ${ }^{4}$ The internal jugular vein (IJV) is the main venous drainage pathway for the brain in the supine position, whereas in the upright position, IJVs collapse and the flow shifts to the vertebral veins (VV) and the vertebral venous plexus. ${ }^{5}$ Additional recruitment of further collateral vessels would alleviate the possible flow disruption within the main drainage pathways.

Along these lines, several magnetic resonance imaging (MRI) studies showed that MS patients have increased collateralization when compared to healthy controls (HC).$^{6-8}$ From increased frequency of posterior paraspinal collaterals, ${ }^{9}$ to trends of greater occurrence in non-IJV collaterals, ${ }^{6}$ MS patients exhibit changes of the extracranial vascular system that are not fully understood. Moreover, a recent hemodynamic MRI study that enrolled 276 MS patients and $106 \mathrm{HCs}$ demonstrated that the MS subjects had decreased flow within the IJVs and increased flow in paraspinal collateral veins. $^{7}$ The possible obstruction in flow within the major draining pathways can cause substantial re-direction of flow
Correspondence: Robert Zivadinov, Center for Biomedical Imaging at Clinical Translational Science Institute, Buffalo Neuroimaging Analysis Center, Jacobs School of Medicine and Biomedical Sciences, University at Buffalo, State University of New York, 100 High Street, Buffalo, NY 14203, USA. Tel.: +1.716.859.7040 - Fax: +1.716.859.7066. E-mail: rzivadinov@bnac.net

Key words: Facial vein; brain atrophy; multiple sclerosis; Doppler sonography.

Received for publication: 4 August 2017. Revision received: 8 September 2017. Accepted for publication: 11 September 2017.

This work is licensed under a Creative Commons Attribution 4.0 License (by-nc 4.0).

C Copyright D. Jakimovski et al., 2017

Licensee PAGEPress, Italy

Veins and Lymphatics 2017; 6:6976

doi:10.4081/vl.2017.6976

toward the anterior/external jugular veins, facial vein (FV), thyroid veins and the vertebral system. The FV is formed by union of the anterior FV and the anterior branch of the posterior FV. Inferiorly, the FV empties into the IJV and drains the blood from areas that largely correspond to the arterial territory of the external carotid artery. Additionally, the cavernous sinus represents an anatomical site of communication between the major venous outflow (IJV) and the FV, and therefore, can facilitate compensatory venous redistribution. Furthermore, it was shown that presence of valves in the facial and ophthalmic veins can regulate bidirectional flow opposing gravitational flow. ${ }^{10}$ The distribution of valves causes the blood flow from the orbital veins to be directed caudally towards the FV and the IJV. Confirming these assumptions, an additional anatomy-based lumped parameter model of the venous circulation has also shown that any increase in resistance of the main venous drainage can cause retrograde flow changes within the cavernous, inferior, and superior petrosal sinuses. ${ }^{11}$ Similarly, a recent interventional study showed that by restoring the main drainage pathway, the collateral flow decreased from $70 \%$ to $30 \% .^{12}$ More importantly, there was a 13 -fold reduction in ventricular size associated with decrease of collateral flow lower than $20 \% .{ }^{12}$ Additionally, it was shown that the presence of venous abnormalities is associated with decreased perfusion in the gray matter (GM), the white matter (WM) and changes in cerebrospinal fluid dynamics. ${ }^{13,14}$

Against this background, we hypothe- 
sized that changes in the venous flow of the extra-cranial neck vessels may be associated with worse clinical and MRI-derived measures in patients with MS. The ability to examine associations between collateral venous flow patterns like the FV and brain inflammatory and neurodegenerative MRI measures may advance the understanding of the vascular pathology in MS.

\section{Materials and methods}

\section{Subjects}

This study utilized data from an ongoing prospective case-control cardiovascular, environmental and genetic (CEG) study. ${ }^{15}$ The study was approved by the local Institutional Review Board (IRB) and all subjects signed a written informed consent. The inclusion criteria for this sub-study were: i) age range of 18-75 years old; ii) MRI and Doppler examination performed within 30 days of the neurological visit; iii) having an MS diagnosis as defined by the 2010 revised McDonald criteria. ${ }^{16}$ Based on their disease course, the MS patients were classified as relapsing-remitting MS (RRMS) or secondary-progressive MS (SPMS). Exclusion criteria for this study consisted of presence of clinical relapse or steroid treatment within 30 days of the MRI scan, or being a nursing mother or pregnant woman. Therefore, any differences within the presence of gadolinium-enhancing lesions is not representative and rendered to only asymptomatic appearance. All subjects underwent echo-color Doppler, MRI and full clinical examination. Standard demographic and clinical information was collected, along with assessing Expanded Disability Status Scale (EDSS) by an experienced neurologist.

\section{MRI acquisition and analysis}

All brain scans were acquired on a $3 \mathrm{~T}$ GE Signa Excite HD 12.0 Twin Speed 8channel scanner (General Electric, Milwaukee, WI, USA) using an 8-channel head and neck (HDNV) coil. There were no MRI hardware or software changes during the study. Acquired MRI sequences included axial 3D-spoiled-gradient recalled (SPGR) T1 weighted image (WI), dual fast spin-echo (FSE) T2/proton density (PD) WI, 2D fluid attenuated inversion recovery (FLAIR) and post contrast spin-echo (SE) T1-WI 5 minutes after single $0.1 \mathrm{mmol} / \mathrm{kg}$ gadobutrol injection. The slice thickness was $1 \mathrm{~mm}$ for $3 \mathrm{D}$ sequences and $3 \mathrm{~mm}$ for $2 \mathrm{D}$ sequences. The MRI acquisition protocol was previously described. ${ }^{17}$

MRI-derived inflammatory measures
(T1, T2 and gadolinium-enhancing lesion volume) were obtained using a semi-automated edge detection and contouring/ thresholding technique. ${ }^{18}$ For calculation of whole brain (WB), GM, WM, ventricular cerebrospinal fluid (vCSF), and cortex normalized volumes, SIENAX cross-sectional software tool was used (version 2.6).${ }^{19}$ Prior to SIENAX, lesions were filled to reduce the impact of $\mathrm{T} 1$ hypointensities. Regional tissue-specific normalized volumes of the thalamus, caudate nucleus $(\mathrm{CN})$, putamen, globus pallidus (GP), hippocampus, and amygdala were derived with FMRIB's Integrated Registration and Segmentation Tool (FIRST).

\section{Doppler sonography assessment}

Echo-color Doppler (ECD Esaote Biosound My Lab 25 Gold) equipped with 2.5 and 7.5-10 $\mathrm{MHz}$ transducers (Genoa, Italy) was used for extra cranial examination. For the purpose of examining the IJV, the $7.5 \mathrm{MHz}$ linear probe was used. The subjects were instructed not to reveal their disease status during the procedure. Additional draping from the neck down was applied in order to further eliminate visual cues of disease. Each subject was evaluated by the same blinded technologist (more than 25 years of experience). The blood flow of both IJVs was assessed. Section above and below the entry of the FV into IJV and levels were used as measurement points for IJV respectively. The flow was calculated by multiplying the time average velocity $\left(\mathrm{V}_{\mathrm{MT}}\right)$ over 4 seconds time phase and vein manually drawn CSA on axial view. The $V_{\mathrm{MT}}$ has been carefully calculated using manual correction of the Doppler angle, whereas the manually drawn CSA was performed on color Doppler settings.

$$
V_{M T}=\Sigma V_{i}^{*} \Delta T
$$

Flow $=V_{M T} * C S A$

FV flow was calculated as the difference of the IJV flow measured below and above the entrance of FV. Figure 1 shows the sites of measurements with the FV into the field of view. The step-wise methodology is shown in Figure 2. The final variable used for further analysis was derived by summation of the blood flow measured within both the left and right side and from hereafter mentioned as FV blood flow. If the blood flow measured above the entry of the FV was higher than the flow measured below the entry, the FV blood flow was labeled as retrograde one.

\section{Statistical analysis}

Statistical analyses were performed using SPSS 24.0 (IBM, Armonk, NY, USA). Demographic and clinical characteristics were compared by using $\chi^{2}$ cross tabulation with Yates' correction, Mann-WhitneyWilcoxon test, and Student's $t$-test, as appropriate. In order to determine the difference in MRI-derived inflammatory and neurodegenerative measures between groups, Student's $t$-test was used. Moreover, analysis of covariance (ANCOVA), where FV status was considered a fixed factor, patients' age was considered a covariance factor, and MRI-derived measures the dependent measure, was performed in order to assess the FV status influence on the MRI-derived measures, controlling for possible aging-related effects. Both Kolmogorov-Smirnov and Shapiro-Wilk tests were used to determine normality of all variables used. Additionally, Q-Q plots were used for visual inspection of the possible data skewness. Four measures (vCSF, DGM, thalamus and GP) were not normally distributed; therefore, normalization by logarithmic transformation was performed. For all statistical results, $\mathrm{P}<0.05$ based on two-tailed tests was considered significant.

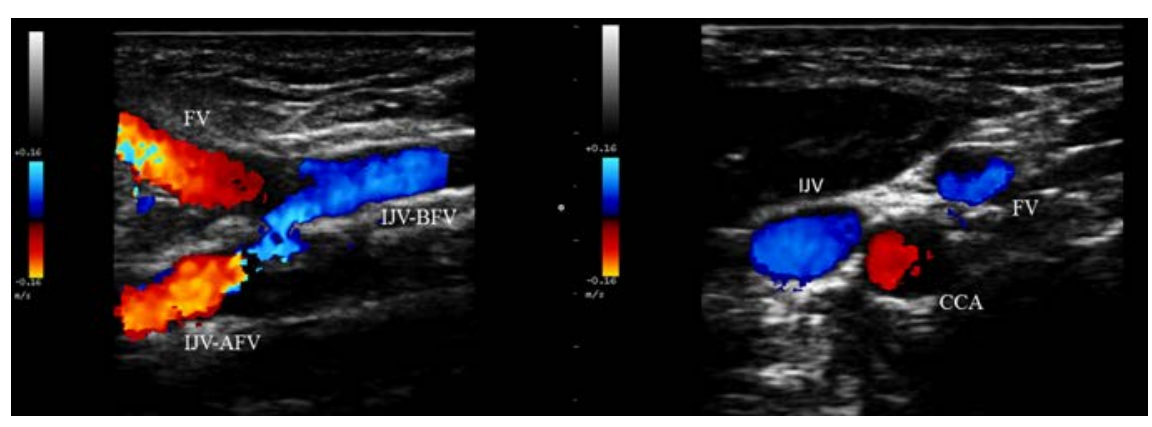

Figure 1. Illustration of the determination of the entrance of the facial vein into the IJV. Sites of measurements above and below the entry were consequently selected. FV, facial vein; IJV, internal jugular vein; AFV, above facial vein; BFV, below facial vein; CCA, common carotid artery. 


\section{Results}

\section{Demographic, clinical, and Doppler} characteristics

The demographic, clinical, and Doppler characteristics of all MS patients $(n=136)$ are summarized in Table 1. The Doppler characteristics of the FV blood flow ranged from $-339.7 \mathrm{~mL} / \mathrm{min}$ to $2229.6 \mathrm{~mL} / \mathrm{min}$. Only 8 MS patients had negative FV blood flow. The mean FV flow was $707.0 \mathrm{~mL} / \mathrm{min}$ and the quartiles were $327.8 \mathrm{~mL} / \mathrm{min}, 651.9$ $\mathrm{mL} / \mathrm{min}$ and, $985.0 \mathrm{~mL} / \mathrm{min}\left(25^{\text {th }}, 50^{\text {th }}\right.$, and, $75^{\text {th }}$, respectively). Additionally, the initial IJV Doppler measurements used for the calculation (TAV, CSA, and flow) are also presented in Table 1. Based on the lowest quartile of FV blood flow $(327.8 \mathrm{~mL} / \mathrm{min})$, the subjects were grouped into higher quartiles and the lowest quartile of FV blood flow (102 vs 34 MS patients, respectively) and hereafter mention as FV status. The higher quartiles group of MS patients, had a mean age of 52.7 years old, a disease duration of 19.6 years, a median disability level of 3.0 EDSS score, and 70 of the patients had the RR form of MS. On the other hand, patients with the lowest quartile of FV blood flow were on average 55.5 years old, had a disease duration of 21.8 years, and had a disability median level based on EDSS scoring of 4.5. The lowest quartile group consisted of proportionally more SPMS patients than the higher quartile MS counterparts $(\mathrm{P}=0.05)$. There were no significant differences in female to male ratio between the groups $(\mathrm{P}=0.13)$. No significant differences between the two MS groups were observed for age $(\mathrm{P}=0.202)$, disease duration
$(\mathrm{P}=0.306)$, EDSS $(\mathrm{P}=0.066)$, and the type of disease modifying therapies used $(\mathrm{P}=0.891)$.

Facial vein blood flow and MRI-derived outcomes

All MRI-derived outcome measures and

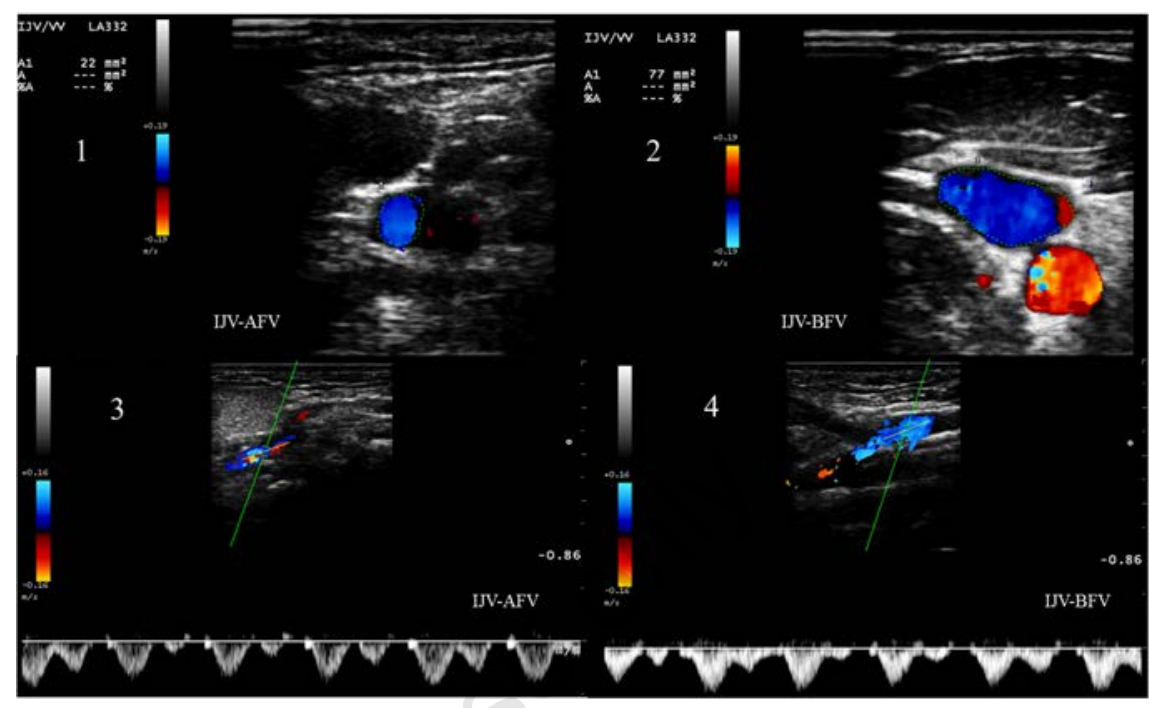

Figure 2. Illustration of the methodology used to measure the facial vein blood flow. IJV, internal jugular vein; AFV, above facial vein; BFV, below facial vein; 1 , cross-sectional area measurement for above the facial vein segment of internal jugular vein; 2 , cross-sectional area measurement for the below facial vein segment below the facial vein; 3 , time-average velocity measurement for above the facial vein segment of internal jugular vein; 4, timeaverage velocity measurement for below the facial vein segment of the internal jugular vein.

Table 1. Demographic and clinical characteristics of the multiple sclerosis cohort.

\begin{tabular}{|c|c|c|c|c|}
\hline Demographic and clinical characteristics & MS cohort $(\mathrm{n}=136)$ & Higher quartiles ( $n=102$ ) & Lowest quartile $(\mathrm{n}=34)$ & P value \\
\hline Female, n (\%) & $100(73.5)$ & $72(70.6)$ & $28(82.4)$ & 0.13 \\
\hline Age in yrs, mean (SD) & $53.4(10.9)$ & $52.7(10.7)$ & $55.5(11.5)$ & 0.202 \\
\hline Disease duration in yrs, mean (SD) & $20.2(10.6)$ & $19.6(10.3)$ & $21.8(11.4)$ & 0.306 \\
\hline $\begin{array}{l}\text { Disease course } \\
\text { RR, n (\%) } \\
\text { SP, n (\%) }\end{array}$ & $\begin{array}{l}87(63.9) \\
49(36.1)\end{array}$ & $\begin{array}{l}70(68.6) \\
32(31.4)\end{array}$ & $\begin{array}{l}17(50.0) \\
17(50.0)\end{array}$ & 0.050 \\
\hline EDSS, median (IQR) & $3.0(4.0)$ & $3.0(2.5)$ & $4.5(4.5)$ & 0.066 \\
\hline $\begin{array}{l}\text { Treatment status } \\
\text { IM IFN-beta-la } \\
\text { SC IFN-beta-la } \\
\text { Natalizumab } \\
\text { Glatiramer acetate } \\
\text { Other DMT } \\
\text { No DMT }\end{array}$ & $\begin{array}{c}30(22.1) \\
10(7.6) \\
5(3.7) \\
38(27.9) \\
24(17.6) \\
27(19.9)^{*}\end{array}$ & $\begin{array}{c}21(20.6) \\
7(6.9) \\
3(2.9) \\
30(29.4) \\
21(20.6) \\
18(16.7)^{*}\end{array}$ & $\begin{array}{c}9(26.5) \\
3(8.8) \\
2(5.9) \\
8(23.5) \\
3(8.8) \\
9(26.5)\end{array}$ & 0.891 \\
\hline Facial vein blood flow (mL/min) & $707.0(511.8)$ & $909.3(413.9)$ & $100.2(203.5)$ & $<0.001$ \\
\hline AFV TAV $(\mathrm{cm} / \mathrm{sec})$ & $20.5(8.7)$ & $20.5(8.4)$ & $20.5(9.5)$ & 0.987 \\
\hline $\operatorname{AFV} \operatorname{CSA}\left(\mathrm{mm}^{2}\right)$ & $36.3(17.6)$ & $35.6(16.9)$ & $38.4(19.6)$ & 0.433 \\
\hline AFV Flow (mL/min) & $874.8(438.4)$ & $849.4(413.5)$ & $951.2(505.1)$ & 0.243 \\
\hline BFV TAV $(\mathrm{cm} / \mathrm{sec})$ & $20.1(7.5)$ & $21.1(7.3)$ & $17.1(7.3)$ & 0.006 \\
\hline BFV CSA $\left(\mathrm{mm}^{2}\right)$ & $67.6(29.6)$ & $72.6(28.3)$ & $52.4(28.6)$ & $<0.001$ \\
\hline BFV Flow (mL/min) & $1581.9(621.6)$ & $1758.7(548.9)$ & $1051.4(521.8)$ & $<0.001$ \\
\hline
\end{tabular}

MS, multiple sclerosis; SD, standard deviation; RR, relapsing remitting; SP, secondary progressive; EDSS, Expanded Disability Status Scale; AFV, above the entry of the facial vein; BFV, below the entry of the facial vein; TAV, time-averaged velocity; CSA, cross-sectional area; IQR, interquartile range; IM, intramuscular; SC, subcutaneous; IFN, interferon; DMT, disease modifying therapy. ${ }^{*} 0$ - DMT data was missing for 2 patients. $\chi^{2}$ - test, Student's t-test and Mann-Whitney test were used accordingly. Alpha level of 0.05 was considered as significant, and is shown in italics. 
differences between groups are summarized in Table 2. Additionally, the differences in volumes are graphically represented in Figure 3. The lowest quartile of FV blood flow group had significantly lower global and regional brain volumes compared to MS patients within the higher quartiles. In particular, they had significantly lower WB volume $(\mathrm{P}<0.001)$, WM volume $(\mathrm{P}<0.001)$, GM volume $(\mathrm{P}=0.004)$, cortical volume $(\mathrm{P}=0.004)$, and a trend for higher vCSF volume $(\mathrm{P}=0.051)$. Similarly, they had lower total deep $\mathrm{GM}$ volume $(\mathrm{P}=0.008)$, thalamic
$(\mathrm{P}=0.003), \mathrm{GP}(\mathrm{P}=0.027)$, and hippocampal $(\mathrm{P}=0.005)$ volumes. All findings were confirmed with ANCOVA analyses controlling for age. Even though several deep gray matter structures did not reach statistical significance, their volumes were smaller in the group within the lowest quartile.

There were no statistical differences between the lowest and the higher quartiles of FV blood flow regarding inflammatory outcome measures, such as T2 hyperintense lesion, gadolinium-enhancing, or T1hypointense lesion volumes.
Independently, we conducted a similar statistical analysis on both disease subgroups. The differences in inflammatory and neurodegenerative MRI-derived measures within the RRMS and SPMS patients are shown in Table 3. Although in lesser effect than compared with a whole sample, the differences in brain atrophy still persisted. In both the RRMS and SPMS subgroups, the patients with the lowest quartile of FV blood flow had smaller WBV and WMV ( $\mathrm{P}=0.01, \mathrm{P}=0.012$ for RRMS, and $\mathrm{P}=0.014, \mathrm{P}=0.012$ for SPMS, respectively).

Table 2. MRI differences between multiple sclerosis patients with lowest and the higher quartiles of bilateral facial vein blood flow.

\begin{tabular}{|c|c|c|c|c|}
\hline MRI characteristics & Higher quartiles $(n=102)$ & Lowest quartile (n=34) & P value & ANCOVA age-adjusted \\
\hline T1-LV & $2.4(5.9)$ & $3.6(6.5)$ & 0.343 & 0.381 \\
\hline T2-LV & $14.6(17.9)$ & $15.4(19.4)$ & 0.819 & 0.951 \\
\hline Gd-LV & $0.023(0.2)$ & $0.002(0.02)$ & 0.569 & 0.720 \\
\hline Whole brain volume & $1463.4(87.7)$ & $1394.0(89.9)$ & $<0.001$ & $<0.001$ \\
\hline Grey matter volume & $741.3(59.3)$ & $706.7(61.1)$ & 0.004 & 0.010 \\
\hline White matter volume & $722.1(42.0)$ & $687.3(42.4)$ & $<0.001$ & $<0.001$ \\
\hline Cortical volume & $602.3(47.4)$ & $573.4(48.3)$ & 0.004 & 0.006 \\
\hline Ventricular CSF volume* & $50.8(25.1)$ & $60.4(29.5)$ & 0.051 & 0.103 \\
\hline Deep grey matter volume* & $54.9(6.7)$ & $51.3(7.3)$ & 0.008 & 0.017 \\
\hline Thalamus volume* & $18.2(2.3)$ & $16.8(2.6)$ & 0.003 & 0.008 \\
\hline Caudate volume & $8.2(1.3)$ & $7.8(1.1)$ & 0.101 & 0.191 \\
\hline Putamen volume & $11.7(1.6)$ & $11.1(1.8)$ & 0.096 & 0.177 \\
\hline Pallidal volume* & $4.0(0.8)$ & $3.7(7.2)$ & 0.027 & 0.034 \\
\hline Hippocampus volume & $8.9(1.3)$ & $8.2(1.3)$ & 0.005 & 0.013 \\
\hline Amygdala volume & $2.9(0.5)$ & $2.8(0.5)$ & 0.115 & 0.180 \\
\hline
\end{tabular}

FV, facial vein; CSF, cerebrospinal fluid; LV, lesion volume, Gd, gadolinium. *Logarithmic transformation used. Student's t-test and analysis of covariance (ANCOVA) adjusted for age were used. Alpha level of 0.05 was

Table 3. Differences based on facial vein status in MRI-derived global and regional brain volumes in individually relapsing-remitting and secondary-progressive MS patients.

\begin{tabular}{|c|c|c|c|c|c|c|c|c|}
\hline \multirow[t]{2}{*}{ MRI characteristics } & \multicolumn{2}{|c|}{ RRMS } & \multirow{2}{*}{ P value } & \multirow{2}{*}{$\begin{array}{l}\text { ANCOVA } \\
\text { age- } \\
\text { adjusted }\end{array}$} & \multicolumn{2}{|c|}{ SPMS } & \multirow{2}{*}{ P value } & \multirow{2}{*}{$\begin{array}{c}\text { ANCOVA } \\
\text { age- } \\
\text { adjusted }\end{array}$} \\
\hline & $\begin{array}{l}\text { Higher } \\
\text { quartiles } \\
\text { (n=70) }\end{array}$ & $\begin{array}{l}\text { Lowest } \\
\text { quartile } \\
\text { (n=17) }\end{array}$ & & & $\begin{array}{l}\text { Higher } \\
\text { quartiles } \\
\text { (n=32) }\end{array}$ & $\begin{array}{l}\text { Lowest } \\
\text { quartile } \\
\text { (n=17) }\end{array}$ & & \\
\hline T1-LV & $1.65(3.6)$ & $3.3(6.7)$ & 0.35 & 0.003 & $4.1(8.9)$ & $3.9(6.6)$ & 0.921 & 0.467 \\
\hline T2-LV & $11.6(15.7)$ & $11.1(14.1)$ & 0.91 & 0.527 & $21.1(20.9)$ & $19.8(23.1)$ & 0.834 & 0.732 \\
\hline Gd-LV & $0.03(0.2)$ & $0.006(0.02)$ & 0.694 & 0.001 & $0.003(0.02)$ & 0.0 & 0.471 & 0.999 \\
\hline Whole brain volume & $1488.8(75.5)$ & $1432.8(92.8)$ & 0.01 & 0.065 & $1407.9(88.1)$ & $1355.3(69.9)$ & 0.012 & 0.892 \\
\hline Grey matter volume & $757.5(54.1)$ & $728.0(62.7)$ & 0.054 & 0.015 & $705.9(55.5)$ & $685.4(52.9)$ & 0.217 & 0.832 \\
\hline White matter volume & $731.2(38.3)$ & $704.8(41.9)$ & 0.014 & 0.725 & $702.1(43.5)$ & $669.9(36.1)$ & 0.012 & 0.722 \\
\hline Cortical volume & $614.4(43.3)$ & $588.2(47.9)$ & 0.031 & 0.009 & $575.7(45.5)$ & $558.7(45.3)$ & 0.217 & 0.898 \\
\hline Ventricular CSF volume* & $47.7(24.4)$ & $53.1(21.5)$ & 0.247 & 0.378 & $57.7(25.5)$ & $67.8(34.9)$ & 0.316 & 0.872 \\
\hline Deep grey matter volume* & $56.7(5.8)$ & $53.8(7.6)$ & 0.063 & 0.032 & $51.3(7.1)$ & $48.9(6.4)$ & 0.286 & 0.482 \\
\hline Thalamus volume* & $18.8(2.1)$ & $17.5(2.7)$ & 0.020 & 0.118 & $16.9(2.4)$ & $16.1(2.3)$ & 0.307 & 0.690 \\
\hline Caudate volume & $8.4(1.1)$ & $8.1(1.2)$ & 0.311 & 0.119 & $7.6(1.3)$ & $7.4(0.9)$ & 0.669 & 0.234 \\
\hline Putamen volume & $12.1(1.5)$ & $11.9(1.8)$ & 0.666 & 0.007 & $10.9(1.6)$ & $10.4(1.6)$ & 0.300 & 0.757 \\
\hline Pallidal volume* & $4.1(0.7)$ & $3.8(7.6)$ & 0.046 & 0.407 & $3.9(0.9)$ & $3.7(0.7)$ & 0.474 & 0.762 \\
\hline Hippocampus volume & $9.1(1.3)$ & $8.6(1.3)$ & 0.139 & 0.044 & $8.5(1.3)$ & $7.7(1.3)$ & 0.071 & 0.306 \\
\hline Amygdala volume & $3.1(0.4)$ & $2.8(0.6)$ & 0.015 & 0.511 & $2.7(0.5)$ & $2.9(0.5)$ & 0.386 & 0.085 \\
\hline
\end{tabular}

RRMS, relapsing-remitting multiple sclerosis; SPMS, secondary progressive multiple sclerosis; FV, facial vein; CSF, cerebrospinal fluid; LV, lesion volume, Gd, gadolinium. *Logarithmic transformation used. Student's ttest and analysis of covariance (ANCOVA) adjusted for age were used. Alpha level of 0.05 was considered as significant, and is shown in italics. The volumes are represented in milliliters (mean \pm standard deviation). 


\section{Discussion}

We evaluated the relationship between collateral venous outflow and MRI-derived outcome measures in MS patients. This study suggests that MS patients within the lowest quartile of FV blood flow presented with decreased global and regional MRIderived brain volumes. Additionally, the lowest quartile group was no different in terms of inflammatory MRI-derived outcome measures when compared to the higher quartile groups.

Studies have shown that MS patients have larger measured arterial inflow than venous drainage, also known as a mismatch between the arterial and venous flow, which may indicate that the venous blood is drained through supplementary, non-previously detected smaller veins. ${ }^{20}$ Additionally, in a global mathematical model, the effect of extra cranial obstacles has been calculated to increase the intracranial pressure, consequently causing a flow reduction up to $70 \%$ in the primary affected vessel, and flow increase in the collateral pathways. ${ }^{21}$ One way of explaining the results of our study is that due to possible obstruction within the IJV, a redistribution of the blood flow through the FV has occurred. The cavernous sinus, as an anatomical communication between the major venous outflow (IJV) and the FV, may facilitate this compensatory mechanism.

Therefore, due to the venous abnormalities, compensatory physiological mechanisms of increased collateralization in MS have been hypothesized. Increased IJV flattening in MS patients was associated with development of more non-IJV collaterals. ${ }^{6}$ Similarly, this morphological development of collateral vessels has been confirmed with corresponding hemodynamic analysis. A recent phase-contrast MR study showed that MS patients had increased quantified paraspinal and collateral veins flow, coupled with reduced blood flow in the IJV. ${ }^{7}$ On the other hand, by using quantitative Doppler ultrasound measurements of the venous blood flow, several studies showed global venous hemodynamic differences associated with MS. A contrast-enhanced ultrasonography study showed heterogeneity in the venous outflow system consistent with slow washout dynamic. ${ }^{22}$

All the aforementioned changes have been previously described as part of chronic cerebrospinal venous insufficiency (CCSVI) condition which is characterized by anomalies of the main extracranial cerebrospinal venous routes that interfere with normal blood outflow. ${ }^{2}$ Higher prevalence of CCSVI has been reported not only in MS patients, but also in Parkinson disease, ${ }^{24}$ and Meniere disease. ${ }^{25}$ Other entities like transient global amnesia and chronic migraine have also been associated with jugular reflux and changes in the venous dynamics. ${ }^{26,27}$ Additionally, well-known MS susceptibility factors like cardiovascular, infectious, and inflammatory risks have been associated with increased prevalence of CCSVI. ${ }^{28}$

The paucity of differences in any of the conventional inflammatory outcome measures may be explained by the recent findings, which suggest that the cortical atrophy, and specifically cortical hemispheric volume loss lateralization, could influence the homeostasis of autonomic nervous system (ANS). ${ }^{29}$ For example, autonomic mechanisms related to cardiovascular control are located in the neuronal circuitry of the insular cortex, dorsal anterior cingulate, prefrontal cortex, and hippocampus. ${ }^{30}$ It has been reported that dysregulation of the ANS is associated with variability of the heart rate, and fluctuations of the blood pressure, all of which can contribute to reaching a critical closing pressure that leads to the collapse of the cerebral venous system. ${ }^{31}$ Therefore, it may be hypothesized that an extensive neurodegenerative pathology within cortical regions associated with ANS system function can alter regional venous flow redistribution. The change of the pos- ture and the change of the physical forces acting on the vasculature are creating normal physiological shift from the IJV-driven venous outflow in supine position to more vertebral and paraspinal flow in erect positon. The initial reports of MS patients having increased IJV venous return in the seated position were supported by other independent studies. ${ }^{23}$ On this basis, a quantitative evaluation study that enrolled patients with RRMS and primary-progressive (PP) MS, and healthy controls showed that the postural dependency was pronounced in the more disabled patients. In that study, $52.9 \%$ of RRMS and $75.9 \%$ of PPMS versus only $13.4 \%$ of HCs showed increased supine IJV flow. ${ }^{32}$ Additionally, this alteration of venous blood outflow was able to discern MS patients against other neurological diseases and versus HCs. ${ }^{33}$

Both concepts of i) lack of differences in inflammatory MRI-derived measures; and ii) increased prevalence of hemodynamic changes within more disabled MS patients (RR vs SP), further converge on the neurodegenerative etiology of the hemodynamic flow changes seen in MS.

Lastly, this study extends the need of more comprehensive vascular analysis. Examining the IJVs only, on several predetermined levels of measurement might not be sufficient in order to detect the possible global hemodynamic changes. We
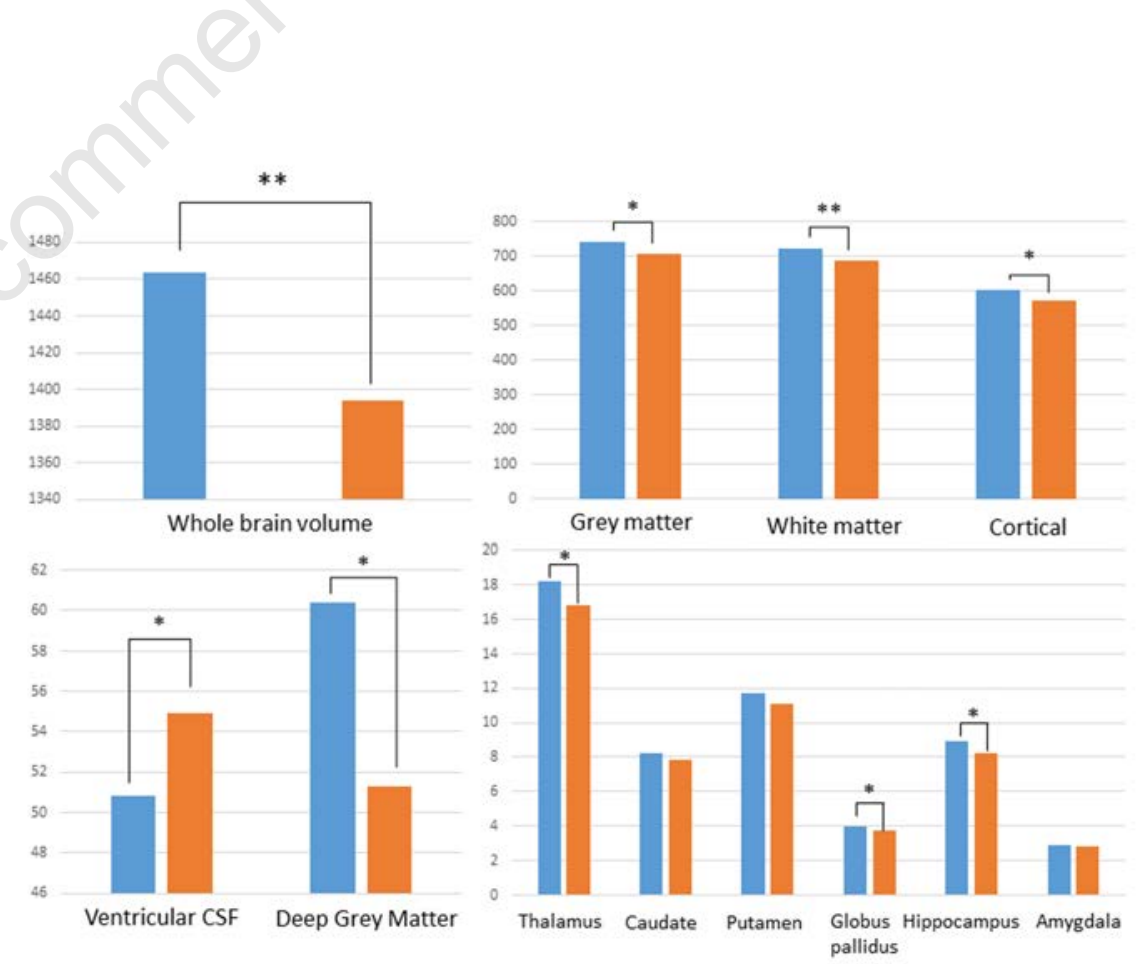

Figure 3. Graphical representation of the differences in brain volumes between groups of lowest quartile and higher quartiles of facial vein blood flow. Orange, multiple sclerosis patients with lowest quartile of facial vein blood flow; blue, multiple sclerosis patients with higher quartiles of facial vein blood flow. ${ }^{*}$ both significant at level of $<\mathbf{0 . 0 5}$; ${ }^{* *}$ correlation significant at level of $<\mathbf{0 . 0 0 1}$. 
showed that flow measured within collateral vessels were able to differentiate patients based on their neurodegenerative phenotype. Well-designed, longitudinal studies comprehensively examining the secondary vascular system and its associations with clinical/MRI measures may overcome the contradictory results reported in the literature.

Despite recent improvements of the pulse-wave Doppler ultrasound, a number of technical limitations in flow measurements still remain inherent to the technique. Conventional ultrasound transducers produce an intensity distribution, which varies continuously across the beam, and conventional pulsed Doppler systems are designed to achieve high spatial resolution rather than uniformity of insonification. Additionally, the method employed also assumes several factors as i) accurate measurement of the CSA, ii) non-turbulent flow, iii) correct angle of insonation, and iv) cylindrically symmetric flow profile. Possible use of newly developed 3D/4D probes that acquire multivolume color Doppler data might circumvent the previously mentioned limitations. Until further standardization of the methods used is achieved, any experimental assessment should serve as a research tool, which might help disentangle the vascular pathology seen in MS patients.

A limitation in this study was the indirect measurement of the FV blood outflow. Color Doppler ultrasound can detect extracranial collateral veins; however, it has limited ability in fully following the complete course of smaller size vessels. Additionally, the available ultrasound flowoutflow data has already been acquired, and therefore our hypothesis was tested in a post-hoc manner. With the FV used as an anatomical marker for the IJV flow measurements, the flow difference between the below and above the entrance of facial vein IJV segment can only be attributed to the actual FV blood flow. In order to determine if the increased collateral flow within the FV has primary pathology or it is a byproduct by the associated neurodegeneration, a prospective Doppler-MRI study is needed.

\section{Conclusions}

MS patients within the lowest quartile of FV blood flow showed more advanced global and regional brain atrophy. The FV can be a substantial alternative venous draining pathway for the head and neck structures and should be considered in future comprehensive venous examinations.
Furthermore, we showed that the ability of the FV to compensate and contribute into the venous drainage is associated with higher global and regional brain volumes. The lack of associations between inflammatory MRI measures in MS patients, but an association with brain atrophy, suggests that the severity of neurodegenerative process may be related to hemodynamic alterations.

\section{References}

1. Zivadinov R, Havrdova E, Bergsland N, et al. Thalamic atrophy is associated with development of clinically definite multiple sclerosis. Radiology 2013;268:831-41.

2. Zamboni P, Galeotti R, Menegatti E, et al. Chronic cerebrospinal venous insufficiency in patients with multiple sclerosis. J Neurol Neurosurg Psychiatry 2009;80:392-9.

3. Dolic K, Siddiqui AH, Karmon Y, et al. The role of noninvasive and invasive diagnostic imaging techniques for detection of extra-cranial venous system anomalies and developmental variants. BMC Med 2013;11:155.

4. Zivadinov R, Bastianello S, Dake MD, et al. Recommendations for multimodal noninvasive and invasive screening for detection of extracranial venous abnormalities indicative of chronic cerebrospinal venous insufficiency: a position statement of the International Society for Neurovascular Disease. J Vasc Interv Radiol 2014;25:1785-94.

5. Ciuti G, Righi D, Forzoni L, et al. Differences between internal jugular vein and vertebral vein flow examined in real time with the use of multigate ultrasound color Doppler. AJNR Am J Neuroradiol 2013;34:2000-4.

6. McTaggart RA, Fischbein NJ, Elkins $\mathrm{CJ}$, et al. Extracranial venous drainage patterns in patients with multiple sclerosis and healthy controls. AJNR Am J Neuroradiol 2012;33:1615-20.

7. Sethi SK, Daugherty AM, Gadda G, et al. Jugular Anomalies in Multiple Sclerosis Are Associated with Increased Collateral Venous Flow. AJNR Am J Neuroradiol 2017 [Epub ahead of print].

8. Zivadinov R, Lopez-Soriano A, Weinstock-Guttman B, et al. Use of MR venography for characterization of the extracranial venous system in patients with multiple sclerosis and healthy control subjects. Radiology 2011;258:56270.

9. Zaharchuk G, Fischbein NJ, Rosenberg
$\mathrm{J}$, et al. Comparison of MR and contrast venography of the cervical venous system in multiple sclerosis. AJNR Am J Neuroradiol 2011;32:1482-9.

10. Zhang J, Stringer MD. Ophthalmic and facial veins are not valveless. Clin Exp Ophthalmol 2010;38:502-10.

11. Marcotti S, Marchetti L, Cecconi P, et al. An anatomy-based lumped parameter model of cerebrospinal venous circulation: can an extracranial anatomical change impact intracranial hemodynamics? BMC Neurol 2015;15:95.

12. Zamboni P, Menegatti E, Cittanti C, et al. Fixing the jugular flow reduces ventricle volume and improves brain perfusion. J Vasc Surg Venous Lymphat Disord 2016;4:434-45.

13. Zamboni P, Menegatti E, WeinstockGuttman B, et al. Hypoperfusion of brain parenchyma is associated with the severity of chronic cerebrospinal venous insufficiency in patients with multiple sclerosis: a cross-sectional preliminary report. BMC Med 2011;9:22.

14. Lagana MM, Chaudhary A, Balagurunathan D, et al. Cerebrospinal fluid flow dynamics in multiple sclerosis patients through phase contrast magnetic resonance imaging. Curr Neurovasc Res 2014;11:349-58.

15. Zivadinov R, Ramasamy DP, Vaneckova M, et al. Leptomeningeal contrast enhancement is associated with progression of cortical atrophy in MS: A retrospective, pilot, observational longitudinal study. Mult Scler 2016:1352458516678083.

16. Polman CH, Reingold SC, Banwell B, et al. Diagnostic criteria for multiple sclerosis: 2010 revisions to the McDonald criteria. Ann Neurol 2011; 69:292-302.

17. Zivadinov R, Heininen-Brown M, Schirda CV, et al. Abnormal subcortical deep-gray matter susceptibility-weighted imaging filtered phase measurements in patients with multiple sclerosis: a case-control study. Neuroimage 2012;59:331-9.

18. Zivadinov R, Rudick RA, De Masi R, et al. Effects of IV methylprednisolone on brain atrophy in relapsing-remitting MS. Neurology 2001;57:1239-47.

19. Smith SM, Zhang Y, Jenkinson M, et al. Accurate, robust, and automated longitudinal and cross-sectional brain change analysis. Neuroimage 2002;17:479-89.

20. Haacke EM, Feng W, Utriainen D, et al. Patients with multiple sclerosis with structural venous abnormalities on MR imaging exhibit an abnormal flow distribution of the internal jugular veins. J Vasc Interv Radiol 2012;23:60-8. 
21. Muller LO, Toro EF, Haacke EM, Utriainen D. Impact of CCSVI on cerebral haemodynamics: a mathematical study using MRI angiographic and flow data. Phlebology 2016;31:305-24.

22. Mancini M, Lanzillo R, Liuzzi R, et al. Internal jugular vein blood flow in multiple sclerosis patients and matched controls. PLoS One 2014;9:e92730.

23. Monti L, Menci E, Ulivelli M, et al. Quantitative ColourDoppler Sonography evaluation of cerebral venous outflow: a comparative study between patients with multiple sclerosis and controls. PLoS One 2011;6:e25012.

24. Liu M, Xu H, Wang Y, et al. Patterns of chronic venous insufficiency in the dural sinuses and extracranial draining veins and their relationship with white matter hyperintensities for patients with Parkinson's disease. J Vasc Surg 2015;61:1511-20.

25. Filipo R, Ciciarello F, Attanasio G, et al.
Chronic cerebrospinal venous insufficiency in patients with Meniere's disease. Eur Arch Otorhinolaryngol 2015;272:77-82.

26. Ertl-Wagner B, Koerte I, Kumpfel T, et al. Non-specific alterations of craniocervical venous drainage in multiple sclerosis revealed by cardiac-gated phase-contrast MRI. Mult Scler 2012;18:1000-7.

27. Chung CP, Hsu HY, Chao AC, et al. Detection of intracranial venous reflux in patients of transient global amnesia. Neurology 2006;66:1873-7.

28. Dolic K, Weinstock-Guttman B, Marr $\mathrm{K}$, et al. Risk factors for chronic cerebrospinal venous insufficiency (CCSVI) in a large cohort of volunteers. PLoS One 2011;6:e28062.

29. Guo CC, Sturm VE, Zhou J, et al. Dominant hemisphere lateralization of cortical parasympathetic control as revealed by frontotemporal dementia.
Proc Natl Acad Sci U S A 2016;113: E2430-2439.

30. Shoemaker JK, Norton KN, Baker J, Luchyshyn T. Forebrain organization for autonomic cardiovascular control. Auton Neurosci 2015;188:5-9.

31. Czosnyka M, Smielewski P, Piechnik S, et al. Critical closing pressure in cerebrovascular circulation. J Neurol Neurosurg Psychiatry 1999;66:606-11.

32. Marchione P, Morreale M, Giacomini P, et al. Ultrasonographic evaluation of cerebral arterial and venous haemodynamics in multiple sclerosis: a casecontrol study. PLoS One 2014;9: e111486.

33. Monti L, Menci E, Piu P, et al. A sonographic quantitative cutoff value of cerebral venous outflow in neurologic diseases: a blinded study of 115 subjects. AJNR Am J Neuroradiol 2014; 35:1381-6. 